\section{Endemic Wild Ornamental Plants from Northwestern Yunnan, China}

\author{
Xiao-Xian Li and Zhe-Kun Zhou ${ }^{1}$ \\ Kunming Institute of Botany, Chinese Academy of Sciences, Kunming, P.R. \\ China 650204
}

\section{Additional index words. horticultural potential}

\begin{abstract}
Northwestern Yunnan is situated in the southern part of the Hengduan Mountains, which is a complex and varied natural environment. Consequently, this region supports a great diversity of endemic plants. Using field investigation in combination with analysis of relevant literature and available data, this paper presents a regional ethnobotanical study of this area. Results indicated that northwestern Yunnan has an abundance of wild ornamental plants: this study identified 262 endemic species (belonging to 64 genera and 28 families) with potential ornamental value. The distinguishing features of these wild plants, their characteristics and habitats are analyzed; the ornamental potential of most plants stems from their wildflowers, but some species also have ornamental fruits and foliage. Among the endemic genera, Pedicularis and Rhododendron have particularly high numbers of ornamental wild species, while Aconitum, Gentiana, Corydalis, Silene, Delphinium, Cremanthodium, and Saussurea also contain significant numbers of wild ornamental species. It is suggested that cultivation of these species may be beneficial, both commercially and to help conserve endangered endemic plant species.
\end{abstract}

Northwestern Yunnan, in southwestern China is a diverse region that is internationally recognized as biodiversity hotspot and a center of plant diversity (Davis et al., 1995; Li, 1994; Mittermeier et al., 2004). Bordering the Tibetan Plateau and northern Myanmar, northwestern Yunnan contains a diversity of vegetation zones spanning subtropical to alpine. As a result of these unique habitats and its location, northwestern Yunnan is characterized by exceedingly high levels of species endemism and harbors an exceptionally high number of rare and vulnerable species.

The variety of habitats in northwestern Yunnan stems from unique landscape features: the region is comprised of rugged, parallel mountain ranges (with glaciated peaks exceeding $6,500 \mathrm{~m}$ ) and deep gorges (down to altitudes of $600 \mathrm{~m}$ ). The mountain ranges provide dispersal corridors for upland flora from the Tibetan Plateau to the northwest and the Sichuan Highlands to the northeast, while the river valleys are conduits for flora from the Indo-Malayan and east Asian lowlands to the southwest and southeast (Sun, 2002). However, the same mountain ranges and gorges that have allowed adjacent floras to overlap, have also maintained sufficient isolation between valleys to enable significant in situ evolution.

Northwestern Yunnan is part of the Mountains of Southwest China Biodiversity Hotspot, which includes the Hengduan, Gaoligong, and $\mathrm{Nu}$ Shan mountains of western Yunnan, and

Received for publication 4 Apr. 2005. Accepted for publication 14 May 2005. Supported by the Natural Science Foundation of Yunnan (grant 2001C0010Z) and the National Key Basic Research Program (973) (grant 2003CB415102). We extend a special thanks to David Melick and Allen Coombes for improving English writing and also for the comments and suggestions and Chen Wen-Yun for his help during the establishment of the database.Z.-K. Zhou appreciates support from JSPS.

1'Author for correspondence; e-mail: zhouzk@mail. kib.ac.cn.

Fig. 1. The study area, northwestern Yunnan of China. have played an important role in world horticulture and have been introduced to Western countries where they have been widely cultivated. Some of the best known examples include Rhododendron, Primula, Gentiana, Pedicularis, and Saussurea, which are all important genera in northwestern Yunnan (Chen et al., 1989; Feng, 1983; Guan et al., 1998; Hu, 1990; Shi and Jin, 1999; Yang, 1956;). Many of these ornamental species are endemic to small areas of northwestern Yunnan (e.g., Rhododendron russatum), therefore, their cultivation not only provides for potential sources of income generation, but also offers a potential form of conservation management: these plants can be used directly for their ornamental plant value or as genetic resources for plant breeding programs. The aims of current paper are to describe the unique flora of northwestern Yunnan and provide detailed information of those resources, in terms of their potential horticultural value as ornamental species.

\section{General Study Area}

Northwestern Yunnan (lat. $25^{\circ} 30^{\prime}-29^{\circ} 15^{\prime} \mathrm{N}$ and long. $98^{\circ} 05^{\prime}-101^{\circ} 15^{\prime} \mathrm{E}$ ) is on the YunnanGuizhou Plateau in southwestern China (Fig. 1). To the west the region merges into the Himalayan mountain ranges, to the north onto the Tibetan Plateau, while the south borders the temperate and subtropical lowlands of central and southern Yunnan. This mountainous region forms the upper reaches of three major river systems, the $\mathrm{Nu}$ Jiang (Salween River), the Lancang (Mekong), and the Jinsha Jiang (the upper reach of the Yangtze River). A wide range of vegetation types exists including, grassland, cushion-like alpine grasslands, evergreen broadleaf forest, mixed forest, alpine mosaic, deciduous broadleaf forest and

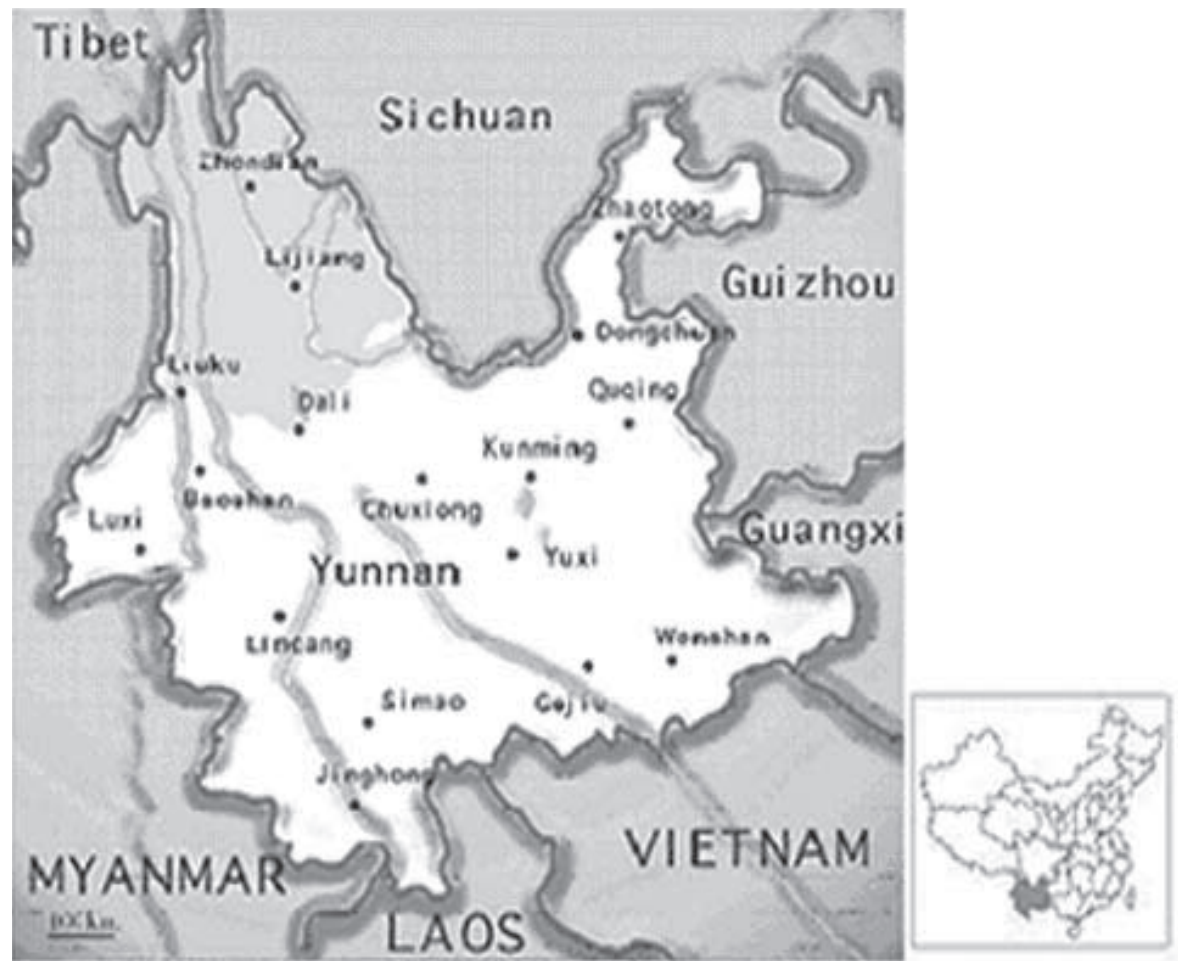


Table 1. The families and genera in order of numbers of endemic wild flower species.

\begin{tabular}{lccc}
\hline Family & No. of species & Genera & No.of species \\
\hline Ranunculaceae & 39 & Pedicularis & 34 \\
Scrophulariaceae & 34 & Rhododendron & 31 \\
Ericaceae & 32 & Aconitum & 23 \\
Compositae & 18 & Gentiana & 16 \\
Gentianaceae & 16 & Corydalis & 16 \\
Rosaceae & 14 & Silene & 13 \\
Caryophyllaceae & 13 & Delphinium & 12 \\
Fumariaceae & 13 & Cremanthodium & 8 \\
Primulaceae & 11 & Saussurea & 8 \\
\hline
\end{tabular}

temperate conifer forest (Wu and $\mathrm{Zhu}, 1987$; Yang, 1990).

The elevations of lower hill areas and valley plains along the Jinsha, Lancang and Nujiang, range between about 700 and $1,100 \mathrm{~m}$. However, the taller mountains, such as Yulong, Haba and Meili snow mountains exceed heights of $5,000 \mathrm{~m}$. The average temperature in this region is 10 to $15^{\circ} \mathrm{C}$, the highest and lowest temperatures in the ranges of 30 to $37^{\circ} \mathrm{C}$ and -4 to $-12{ }^{\circ} \mathrm{C}$, respectively. The annual average rainfall is between 1,185 and $1,439 \mathrm{~mm}$ (Yang, 1990).

Site selection. Northwestern Yunnan covers 16 counties of Diqing, Lijiang, Nujiang, and Dali Prefectures, lat. $25^{\circ} 30^{\prime}-29^{\circ} 15^{\prime} \mathrm{N}$, and long. $98^{\circ} 05^{\prime}-101^{\circ} 15^{\prime} \mathrm{E}$. Its total area is $68,908 \mathrm{~km}^{2}$ (17.5\% of Yunnan Province).

Sites were selected according to the physicogeographical regionalization parameters outlined by Yang (1990) which are comprehensive contributions about physicogeographical regionalization of Yunnan. We used six areas: Yunlong, Lanping alpine-medium hillside area $\left(15,000 \mathrm{~km}^{2}\right)$, Nu Jiang alpine mountains and gorge area $\left(10,000 \mathrm{~km}^{2}\right)$, Dali and Lijiang basin and alpine-medium coteau area $(26,000$ $\left.\mathrm{km}^{2}\right)$, Jinsha Jiang river valley area $(15,000$ $\mathrm{km}^{2}$ ), Zhongdian and Deqin alpine mountains and plateau area $\left(16,000 \mathrm{~km}^{2}\right)$, and part of the Baoshan and Fengqing medium mountain and basin area. In terms of political boundaries, the study sites covered the northern part of the Dali Bai Autonomous Prefecture, most of the Lijiang Naxi Autonomous Prefecture, the Nujiang Lisu Autonomous Prefecture and Diqing Zang Autonomous Prefecture. 16 counties are included in the study area: Yunlong, Dali, Eryuan, Jianchuan, Heqing, Binchuan, Lijiang, Ninglang, Yongsheng, Zhongdian, Deqin, Weixi, Lanping, Lushui, Fugong, and Gongshan (Fig. 1).

\section{Methods}

An initial check list was made of the presence and distribution of plants in the study areas, based on the following plant surveys: List of seed plants in Yunan (Kunming Institute of Botany, 1984), Vegetation of Yunnan (Wu and Zhu, 1987), Vascular Plants in Hengduan Mountains (Chinese Academy of Science, 1993, 1994), Flora of Yunnan (Wu and Chen, 1986, 2000; Wu etal., 1997), and Flora of China (Chen and Hu, 1989; Hu, 1990; Shi and Jin, 1999; Yang, 1956). Herbarium records from the Kunming Institute of Botany were used to validate field samples. The compiled database included the following information: species, genus and family names, plant attributes includ- ing distribution patterns, life form, vegetation ornamental value. Using this database, we identified those endemic species that had ornamental value. Evaluation and reinvestigation of this list of endemic plants from northwestern Yunnan was based on literature, herbarium records, the database and field work.

Definition of ornamental plants. Different botanist have different definitions of ornamental plants. In this study, we consider wild ornamental plants to be those which occur naturally in the field and have highly ornamental features such as ornamental flowers, fruit, or foliage.

\section{Results and Discussion}

Wild ornamental plants endemic to northwestern Yunnan. In total, northwestern Yunnan contains 2,206 species, (belonging to 324 genera and 38 families), of which 910 species (41\%) are endemic (Li et al., 2003). There are a total of nine endemic families with more than eight endemic species. A considerable number of these endemic species were found to be wild flowers: we identified 262 species, belonging to 64 genera and 28 families endemic to northwestern Yunnan. Among the endemic genera, Pedicularis and Rhododendron are particularly rich in endemic wild ornamental species (Table 1).

Analysis of life-form. The inventory shows that most of the endemic wild flowers are herbaceous which probably is th results of the harsh geographical environment in the study area (Table 2). Alpine plants are highly adapted to growth in particularly harsh conditions, since they have evolved special characteristics in response to a particular combination of cool climate and mountainous terrain. The cool climate in the study area, particularly in winter, results in generally low temperatures, frequent frosts and strong winds. Snow persists at the highest altitudes, for 1 to 3 months in the subalpine zone and 3 to 4 months in the alpine zone. These factors have major effects on plant growth. Low temperatures, frosts and snow restrict plant growth for most of the year, particularly at high altitudes. Northwestern Yunnan alpine and mountain areas are characterised by a sequence of vegetation communities with distinct altitudinal zones. Vegetation communities are groups of plants living together with similar preferences and tolerances for particular environmental conditions. These communities can be described by differences in species, height and growth form, and structure. The most obvious change in communities is the transition, at the altitude types, flower colors, flowering season and known as the treeline, to a treeless vegetation. Above the treeline is the true alpine zone where the vegetation is primarily dwarfed shrubs and ground hugging herbs, with no trees.

The growth habit also helps in surviving freezing temperatures: cushion and rosette herbs may be $10^{\circ} \mathrm{C}$ higher than the surrounding air.

Analysis of ornamental features. Ornamental plants can be divided into three main types: those with ornamental flowers, ornamental fruits, or ornamental foliage. However, many wild plants have several ornamental parts, therefore, these divisions serve to distinguish different groups on their primary ornamental features.

The results show that of the endemic ornamental plants in northwestern Yunnan, 235 species have ornamental flowers, and most of them belong to the families Ericaceae, Compositae, Liliaceae, Orchidaceae, Ranunculaceae, Rosaceae, and Gentianaceae. Flower colors vary from red and purple to white and yellow. The red-flowered plants include some species of the genus Pedicularis, such as $P$. habachanensis, $P$. praeruptorum; the genus Rhododendron, such as $R$. cyanocarpum, $R$. fastigiatum, $R$. codonanthum; and the genus Androsace, such as A. alchemilloides. Most of the purple-flowered plants belong to the genera Gentiana, Primula, Aconitum, Delphinium, and Corydalis (exemplified by species such as $G$. bella, P. annulata, A. acutisculum, D. brevisepalum, and $C$. petrophila). White-flowered species include Sorbus harrowiana, Spiraea calcicola, Anemone multilobulata, prominent yellow-green flowers include Rhododendron dendricola, R. rhombifolium, Pedicularis lecomtei, and P. tomentosa.

Table 3 shows the approximate flowering times of 262 wild ornamental plants endemic to northwestern Yunnan. Flowering times are affected by a variety of factors, including, habitat, elevation and species type. Generally at higher elevations plants will bloom later and possibly for longer periods of time. The number of species blooming in early spring and midsummer is greater than those blooming in fall or mid-winter. Most Pedicularis spp., Rhododendron spp., Primula spp. and Gentiana spp. bloom in early spring and midsummer, whereas, Cremanthodium spp. and Delphinium spp. bloom in fall and winter.

There are only 17 endemic wild species with ornamental fruits in northwestern Yunnan. These plants have fruits with a vivid color or unique shape; most of the species belong to Aceraceae, Rosaceae, Aquifoliaceae and Actinidiaceae. Plants from the Aceraceae have light purple or purplish green, winged fruits, each consisting of a pair of samaras which

Table 2. Analysis of the life-form of endemic wild ornamental plants in northwestern Yunnan.

\begin{tabular}{lcc}
\hline & $\begin{array}{c}\text { Endemic wild } \\
\text { ornamental } \\
\text { Life }\end{array}$ & \\
form & (species) & Percentage \\
\hline Herb & 184 & 70.2 \\
Shrub & 66 & 25.2 \\
Tree & 12 & 4.6 \\
\hline
\end{tabular}


Table 3. Inventory of wild ornamental plants endemic to northwestern Yunnan; $\mathrm{Fl}=$ flower, $\mathrm{Fr}=$ fruit, $\mathrm{Fo}=$ foliage

\begin{tabular}{|c|c|c|c|c|}
\hline Species & Life form & Color & Fl or Fr time & Habitat \\
\hline Acer kiukiangense Hu et WC Cheng & Evergreen trees & Fr purplish-green & Fr Sept. & Evergreen broad-leaved forest at $1,700 \sim 2,200 \mathrm{~m}$ \\
\hline Acer lanpingensis Feng et Fang & Deciduous trees & Fr light blue & Fr Sept. & Evergreen broad-leaved forest at 2,600 2,700 m \\
\hline Acer machilifolium $\mathrm{Hu}$ et Cheng & Evergreen trees & Fr purplish-green & Fr Jan.-Feb. & Evergreen broad-leaved forest at $1,600 \sim 2,400 \mathrm{~m}$ \\
\hline Aconitum acutiusculum Fletcher et Lauener & Perennial herbs & Fl purplish-blue & Fl Aug. & Alpine meadow,alpine gravel desert at 4,000 m \\
\hline Aconitum bisma (Buch.-Ham.) Rapaices & Perennial herbs & Fl light yellow & Fl Aug.-Sept. & Alpine meadow at $3,400 \sim 3,800 \mathrm{~m}$ \\
\hline Aconitum bracteolosum WT Wang & Perennial herbs & Fl bluish-purple & Fl July & Alpine meadow at $4,800 \mathrm{~m}$ \\
\hline Aconitum brevipetalum WT Wang & Perennial herbs & Fl blue & Fl Aug.-Oct. & Alpine meadow at $2,800 \sim 3,800 \mathrm{~m}$ \\
\hline Aconitum chuianum WT Wang & Perennial herbs & Fl deep blue & Fl Aug. & Alpine meadow at $2,800 \sim 3,800 \mathrm{~m}$ \\
\hline Aconitum coriophyllum Hand.-Mazz & Perennial herbs & Fl greenish-yellow & Fl Oct. & Alpine meadow at 2,600 m \\
\hline Aconitum crassiflorum Hand.-Mazz. & Perennial herbs & Fl bluish-purple & Fl July-Aug. & Alpine meadow or fir forest at $3,600 \sim 4,100 \mathrm{~m}$ \\
\hline Aconitum delavayi $\mathrm{Fr}$ & Perennial herbs & Fl bluish-purple & Fl Aug.-Oct. & Alpine meadow at $3,700 \sim 3,800 \mathrm{~m}$ \\
\hline Aconitum dolichorhynchum WT Wang & Perennial herbs & Fl bluish-purple & Fl Aug. & Alpine shrubs and meadow at $3,400 \mathrm{~m}$ \\
\hline Aconitum duclouxii H. Lév & Perennial herbs & Fl bluish-purple & Fl Sept. & Alpine meadow or fir fores at $3,600 \sim 4,100 \mathrm{~m}$ \\
\hline Aconitum georgei HF Comber & Perennial herbs & Fl bluish-purple & Fl July-Sept. & Alpine shrubs and meadow at $3,700 \sim 4,000 \mathrm{~m}$ \\
\hline Aconitum habaense WT Wang & Perennial herbs & Fl bluish-purple & Fl Aug. & Alpine shrubs at about $3,600 \mathrm{~m}$ \\
\hline Aconitum kungshanense WT Wang & Perennial herbs & Fl bluish-purple & Fl Aug.-Sept. & Alpine rhododendron shrubs at $3,400 \sim 4,100 \mathrm{~m}$ \\
\hline Aconitum incisofidum W.T. Wang & Perennial herbs & Fl purple & Fl Aug.-Sept. & Alpine shrubs at 3,700 3,900 m \\
\hline Aconitum pseudostapfianum WT Wang & Perennial herbs & Fl blue & Fl Aug. & Alpine meadow at about $4,150 \mathrm{~m}$ \\
\hline Aconitum ramulosum WT Wang & Perennial herbs & Fl bluish-purple & Fl July-Aug. & Alpine shrubs and meadow at $3,700 \sim 4,000 \mathrm{~m}$ \\
\hline Aconitum souliei Finet et Gagnepain & Perennial herbs & Fl yellow & Fl Aug. & Alpine meadow at 3,800 3,900 m \\
\hline Aconitum stramineiflorum Chang ex WT Wang & Perennial herbs & Fl yellow & Fl Aug. & Alpine meadow at $3,900 \mathrm{~m}$ \\
\hline Aconitum taronense (Hand.-Mazz.) Fletcher et Lauener & Perennial herbs & Fl purple & Fl Aug.-Sept. & Alpine meadow at 2,600 3,600 m \\
\hline Aconitum tenuicaule WT Wang & Perennial herbs & Fl bluish-purple & Fl Aug. & Alpine meadow, gravel desert at $4,000 \mathrm{~m}$ \\
\hline Aconitum tsaii WT Wang & Perennial herbs & Fl blue & Fl Aug.-Sept. & Subalpine evergreen broad-leaved forest at 2,000 3,600 m \\
\hline Aconitum weixiense WT Wang & Perennial herbs & Fl blue & Fl Aug.-Sept. & Subalpine evergreen broad-leaved forest at $2,000 \sim 3,600 \mathrm{~m}$ \\
\hline Aconitum yangii WT Wang et $\mathrm{LQ} \mathrm{Li}$ & Perennial herbs & Fl blue & Fl Aug. & Alpine meadow at $3,100 \mathrm{~m}$ \\
\hline $\begin{array}{l}\text { Actinidia pilosula (Finet \& Gagnagnepain) } \\
\text { Stapf ex Hand.-Mazz. }\end{array}$ & Woody vines & Fl White, Fr gold-yellow & Fl June, Fr Aug. & Evergreen broad-leaved forest at 2,300 2,400 m \\
\hline Aeschynanthus angustioblongus WT Wang & Climbing shrubs & Fl red & Fl Sept. & Dry-hot valley shrubs at $1,250 \sim 1,500 \mathrm{~m}$ \\
\hline Aeschynanthus lasianthus WT Wang & Climbing shrubs & Fl deep-red & Fl Aug.- Oct. & Dry-hot valley shrubs at $1,600 \sim 2,300 \mathrm{~m}$ \\
\hline Amitostigma trifurcatum T. Tang, FW Wang et KY Lang & Herbs & Fl yellow & Fl June & Evergreen broad-leaved forest at $600 \sim 700 \mathrm{~m}$ \\
\hline Androsace alchemilloides $\mathrm{Fr}$ & Perennial herbs & Fl pink & Fl May- June & Alpine shrubs and meadow at $3,000 \sim 4,000 \mathrm{~m}$ \\
\hline Androsace bulleyana Forr. & Biennial or perennial herbs & Fl pink & Fl June-July & Alpine gravel desert at $3,200-3,900 \mathrm{~m}$ \\
\hline Androsace graceae Forrest & Perennial herbs & Fl pink & Fl June-July & Alpine gravel desert at $3,800 \sim 4,600 \mathrm{~m}$ \\
\hline Anemone multilobulata WT Wang et LQ Li & Perennial herbs & Fl white & Fl Apr--May & Alpine meadow at 3,500 3,800 m \\
\hline Anemone scabriuscula WT Wang & Perennial herbs & Fl white & Fl May-Oct. & Subalpine meadow at about $2,900 \mathrm{~m}$ \\
\hline Aster brevis Hand.-Mazz. & Perennial herbs & Fl purple-red & Fl July-Sept. & Alpine shrubs and meadow at 2,900 3,500 m \\
\hline Aster bulleyanus Jeffrey & Perennial herbs & Fl purple-red & Fl Mar--Oct. & Subalpine shrubs and meadow at $1,100 \sim 3,500 \mathrm{~m}$ \\
\hline Berberis amoena Dunn. & Shrubs & Fl yellow, Fr red & Fl Nov.-Dec., Fr June-Aug. & Subalpine shrubs and meadow at 2,100 3,000 m \\
\hline Berberis calcipratorum Ahrendt & Shrubs & Fl dark-yellow, Fr yellow & Fl June, Fr Aug. & Alpine shrubs and meadow at $3,000 \sim 4,000 \mathrm{~m}$ \\
\hline Berberis concolor WW Sm. & Shrubs & Fl yellow, Fr purplish-red & Fl May-June, Fr July-Aug. & Alpine shrubs and meadow at 2,900 3,600 m \\
\hline Berberis forrestii Ahrendt & Shrubs & Fl yellow, Fr bright red & Fl June, Fr Oct. & Alpine shrubs at 2,800 3,600 m \\
\hline Berberis minutiflora Schneid. & Shrubs & Fl yellow, Fr red & Fl May-June, Fr Sept.-Oct. & Alpine shrubs at 2,500 3,600 m \\
\hline Carissa yunnanensis Tsiang et PT Li & Evergreen shrubs & Fl white & Fl Apr. & Evergreen broad-leaved forest at $1,200 \sim 1,800 \mathrm{~m}$ \\
\hline Cassiope argyrotricha TZ Hsu & Shrubs & Fl white & Fl July-Aug. & Alpine or subalpine scree slopes at 3,000 3,900 m \\
\hline Cassiope nana TZ Hsu & Dwarf evergreen shrub & Fl milk white & Fl July-Aug. & Alpine or subalpine scree slopes at 3,200 3,900 m \\
\hline Cassiope palpebrata WW Sm. & Shrubs & Fl milk white & Fl July-Aug. & Alpine or subalpine scree slopes at 3,000 3,900 m \\
\hline Cassiope ectinata Stapf & Shrubs & Fl milk white & Fl July-Aug. & Alpine or subalpine scree slopes at 3,300 3,900 m \\
\hline Cephalanthera calcarata SC Chen et KY Lang & Herbs & Fl pink & Fl Sept. & Coniferous and broad-leaved mixed forest at $2,900 \sim 3,000 \mathrm{~m}$ \\
\hline Cheiranthus forrestii (WW Sm.) Hand.-Mazz. & Perennial herbs & Fl purplish-red & Fl June-July & Alpine gravel desert at $4,700 \sim 5,200 \mathrm{~m}$ \\
\hline Clematis venusta $\mathrm{MC}$ Chang & Woody vines & Fl white & Fl May & Coniferous and broad-leaved mixed forest at 2,300 2,700 m \\
\hline Coelogyne corymbosa Lindl. & Adnascent herbs & Fl yellowish-green & Fl Apr.-May & Evergreen broad-leaved forest at 1,150 1,500 m \\
\hline Corallodiscus patens (Craib) BL Burtt & Perennial herbs & Fl purplish-blue & Fl July-Aug. & Alpine meadow at 3,000 3,300 m \\
\hline Corydalis bijiangensis $\mathrm{CY}$ Wu et $\mathrm{H}$. Chuang & Perennial herbs & Fl yellow & Fl July & Alpine meadow,alpine gravel desert at $4,200 \sim 4,600 \mathrm{~m}$ \\
\hline Corydalis calcicola WW Sm & Perennial tufty herbs & Fl yellow & Fl July & Alpine gravel desert at 3,800 4,200 m \\
\hline Corydalis cheirifolia $\mathrm{Fr}$ & Perennial tufty herbs & Fl yellow & Fl July-Nov. & Subalpine meadow at 2,100 2,500 m \\
\hline Corydalis delavayi $\mathrm{Fr}$ & Perennial herbs & Fl blue & Fl July-Nov. & Alpine gravel desert at $3,800 \sim 4,200 \mathrm{~m}$ \\
\hline Corydalis hemidicentra Hand.-Mazz. & Perennial herbs & Fl bluish & Fl July-Nov. & Alpine gravel desert at $3,500 \sim 4,100 \mathrm{~m}$ \\
\hline Corydalis heterocentra Diels & Perennial herbs & Fl blue & Fl July-Nov. & Scree slopes at 3,900 4,500 m \\
\hline Corydalis lopinensis $\mathrm{Fr}$ & Perennial tufty herbs & Fl yellow & Fl May-June & Subalpine meadow at $1,350 \sim 3,900 \mathrm{~m}$ \\
\hline Corydalis mayae Hand.-Mazz. & Perennial herbs & Fl yellow & Fl May-June & Alpine shrubs, meadow at $3,800 \sim 4,200 \mathrm{~m}$ \\
\hline Corydalis multisecta CY Wu et $\mathrm{H}$. Chuang & Perennial herbs & Fl pale blue, purple & Fl July & Dry ravine on steep slope under shrubs at $4,100 \sim 4,300 \mathrm{~m}$ \\
\hline Corydalis oxypetala $\mathrm{Fr}$ & Perennial herbs & Fl yellow & Fl May-July & Limestone shrubs and meadow at 3,500 3,600 m \\
\hline Corydalis petrophila $\mathrm{Fr}$ & Perennial herbs & Fl purple & Fl July-Sept. & Alpine meadow at 3,000 3,400 m \\
\hline Corydalis atuntsiensis WW Sm. & Perennial herbs & Fl pale violet & Fl July & Alpine shrubs, meadow at $3,800 \sim 4,200 \mathrm{~m}$ \\
\hline Corylopsis glaucescens Hand.-Mazz. & Shrubs & Fl yellowish-green & Fl Mar--Apr. & Coniferous and broad-leaved mixed forest at $1,700 \sim 3,100 \mathrm{~m}$ \\
\hline Corylopsis trabeculosa He et Cheng & Shrubs & Fl yellowish-green & Fl after Oct. & Coniferous and broad-leaved mixed forest at 1,350 2,000 m \\
\hline Corylopsis yui Hu et WC Cheng & Shrubs & Fl yellowish-green & Fl Aug.- Oct. & Evergreen broad-leaved forest at $2700 \sim 3600 \mathrm{~m}$ \\
\hline Corylopsis yunnanensis Diels. & Shrubs & Fl yellowish-green & Fl Mar--Apr. & Coniferous and broad-leaved mixed forest at $2,400 \sim 2,800 \mathrm{~m}$ \\
\hline Cotinus nana WW Sm. & Shrubs, small trees & Fo red & Fo Oct--Dec. & Dry-hot valley shrubs at $600 \sim 1,500 \mathrm{~m}$ \\
\hline Crataegus chungtienensis WW Sm. & Shrubs & Fl white, Fr red & Fl May & Alpine shrubs and meadow at 2,800 3,280 m \\
\hline Crataegus oresbia WW Sm. & Shrubs & Fl white, Fr red & Fl May & Alpine shrubs at $3,000 \sim 3,300 \mathrm{~m}$ \\
\hline Cremanthodium glaucum Hand.-Mazz. & Perennial herbs & Fl yellow & Fl May-Sept. & Alpine rhododendron shrubs at $3,900 \sim 4,300 \mathrm{~m}$ \\
\hline Cremanthodium brachychaetum CC Chang & Perennial herbs & Fl yellow & Fl July-Aug. & Alpine shrubs and meadow at $3,100 \sim 3,600 \mathrm{~m}$ \\
\hline Cremanthodium calcicola WW Sm. & Perennial herbs & Fl yellow & Fl July-Oct. & Alpine meadow at 2,500 3,300 m \\
\hline Cremanthodium chungtienense Ling et SW Liu & Perennial herbs & Fl yellow & Fl Aug.-Oct. & Alpine rhododendron shrubs at 3,200 3,300 m \\
\hline Cremanthodium delavayi Fr et Diels ex H. Léveillé & Perennial herbs & Fl yellow & Fl July-Aug. & Alpine rhododendron shrubs at $3,200 \sim 4,000 \mathrm{~m}$ \\
\hline Cremanthodium dissectum Grierson & Perennial herbs & Fl yellow & Fl July-Aug. & Rhododendron shrubs, alpine shrubs, meadow at $2,295 \sim 4,800 \mathrm{~m}$ \\
\hline Cremanthodium farreri WW Sm. & Perennial herbs & Fl yellow & Fl July-Sept. & Alpine rhododendron shrubs, gravel desert at 2,950 4,000 m \\
\hline Cremanthodium pulchrum RD Good & Perennial herbs & Fl purplish-red & Fl Aug.-Oct. & Alpine shrubs and meadow at $3,200 \sim 4,600 \mathrm{~m}$ \\
\hline Cypripedium margaritaceum $\mathrm{Fr}$ & adnascent herbs & Fl yellowish to white & Fl Mar-Apr. & Evergreen broad-leaved forest at $1,600 \sim 1,700 \mathrm{~m}$ \\
\hline Delphinium brevisepalum WT Wang & Perennial herbs & Fl yellow & Fl Jul.-Aug. & Alpine meadow at 2,900 3,800 m \\
\hline Delphinium bulleyanum Forrest ex Diets. & Perennial herbs & Fl blue & Fl Aug.-Sept. & Alpine meadow at $3,100 \sim 3,700 \mathrm{~m}$ \\
\hline Delphinium ceratophorum $\mathrm{Fr}$ & Perennial herbs & Fl bluish-purplish & Fl Aug.-Sept. & Alpine gravel desert, shrubs at 2,400 3,600 m \\
\hline Delphinium coleopodum Hand.-Mazz. & Perennial herbs & Fl blue & Fl Sept.-Oct. & Alpine meadow at 3,600 3,900 m \\
\hline
\end{tabular}




\begin{tabular}{|c|c|c|c|c|}
\hline Species & Life form & Color & Fl or Fr time & Habitat \\
\hline Delphinium hamatum $\mathrm{Fr}$ & Perennial herbs & Fl bluish-purple & Fl Sept-Oct. & Alpine meadow at 2,900 3,800 m \\
\hline Delphinium latirhombicum WT Wang & Perennial herbs & Fl blue & Fl July & Alpine meadow at $2,900 \mathrm{~m}$ \\
\hline Delphinium likiangense $\mathrm{Fr}$ & Perennial herbs & Fl blue & Fl Aug.-Sept. & Alpine meadow or gravel desert at $3,400 \sim 4,500 \mathrm{~m}$ \\
\hline Delphinium majus (WT Wang) WT Wang & Perennial herbs & Fl bluish-purple & Fl Aug.-Sept. & Dry-hot valley shrubs at $1,600 \sim 1,800 \mathrm{~m}$ \\
\hline Delphinium micropetalum Finet etGagnep. & Perennial herbs & Fl bluish-purple & Fl Aug.-Oct. & Alpine meadow at $3,300 \sim 4,200 \mathrm{~m}$ \\
\hline Delphinium pseudohamatum WT Wang & Perennial herbs & Fl blue & Fl Sept. & Alpine meadow at about $3,790 \mathrm{~m}$ \\
\hline Delphinium yangii WT Wang & Perennial herbs & Fl blue & Fl July-Aug. & Alpine shrubs and meadow at $4,200 \sim 4,500 \mathrm{~m}$ \\
\hline Delphinium yulungshanicum WT Wang & Perennial herbs & Fl purplish-blue & Fl Sept.-Oct. & Alpine meadow at 3,300 4,200 m \\
\hline Dolomiaea berardioidea (Fr) C.Shih. & Perennial herbs & Fl purple & Fl Sept. & Alpine gravel desert at $4,500 \sim 4,800 \mathrm{~m}$ \\
\hline Dolomiaea salwinensis (Hand.-Mazz.) C.Shih. & Perennial herbs & Fl purple & Fl Aug.-Oct. & Subalpine shrubs, Rhododendron shrubs at 2,400 4,100 m \\
\hline Draba involucrate WW Sm. & Tufty herbs & Fl yellow & Fl May-July & Alpine shrubs, meadow, gravel desert at 4,050 4,100 m \\
\hline Draba jucunda WW Sm. & Tufty herbs & Fl yellow & Fl June-Aug. & Alpine gravel desert or shrubs and meadow at $4,075 \sim 4,375 \mathrm{~m}$ \\
\hline Draba oreodoxa WW Sm. & Tufty herbs & Fl yellow & Fl May-July & Alpine shrubs and meadow at $2,400 \sim 4,600 \mathrm{~m}$ \\
\hline Draba piepunensis OE Schulz. & Tufty herbs & Fl yellow & Fl July-Aug. & Alpine meadow at $3,700 \sim 4,650 \mathrm{~m}$ \\
\hline Draba senilis OE Schulz. & Perennial herbs & Fl yellow & Fl May-July & Alpine meadow, gravel desert at about $4,000 \mathrm{~m}$ \\
\hline Draba serpens OE Schulz. & Groundling herbs & Fl yellow & Fl June-July & Alpine gravel desert at $4,100 \sim 5,500 \mathrm{~m}$ \\
\hline Dysosma aurantiocaulis (Hand. -Mazz.) Hu & Perennial herbs & Fl dark red & Fl May-June & Evergreen broad-leaved forest at 2,800 3,000 m \\
\hline Eriobotrya tengyuehensis WW Sm. & Evergreen shrubs & Fl milk yellow, Fr red & Fl Apr--May & Subalpine shrubs at $1,700 \sim 2,500 \mathrm{~m}$ \\
\hline Fritillaria crassicaulis S. C. Chen & Perennial herbs & Fl yellowish-green & Fl May & Alpine meadow at $3,000 \sim 3,900 \mathrm{~m}$ \\
\hline Gaultheria cardiosepala Hand.-Mazz. & Shrubs & Fr white & Fr Aug.-Oct. & Scree slopes at 2,800 3,600 m \\
\hline Gentiana alsinoides $\mathrm{Fr}$ & Annual herbs & Fl light blue & Fl July-Sept. & Limestone shrubs at $2,700 \sim 3,350 \mathrm{~m}$ \\
\hline Gentiana anisostemon C. Marquand. & Annual herbs & Fl blueish-purple & Fl Apr--May & Alpine meadow, shrubs at $3,600 \sim 4,300 \mathrm{~m}$ \\
\hline Gentiana asparagoides TN Ho & Annual herbs & Fl bluish-purple & Fl Aug. & Alpine wetland at $3,500 \sim 3,800 \mathrm{~m}$ \\
\hline Gentiana bella $\mathrm{Fr}$ & Annual herbs & Fl bluish-purple & Fl June-Aug. & Alpine meadow at $3,000 \sim 4,050 \mathrm{~m}$ \\
\hline Gentiana caryophyllea Harry Sm. & Perennial herbs & Fl deep blue & Fl Aug.-Sept. & Alpine meadow at $4,000 \sim 4,300 \mathrm{~m}$ \\
\hline Gentiana chungtienensis C. Marquand & Annual herbs & Fl light blue & Fl May-June & Alpine shrubs and meadow at $3,000 \sim 3,700 \mathrm{~m}$ \\
\hline Gentiana dentiformis TN Ho & Annual herbs & Fl yellowish-yellow & Fl Aug. & Deciduous pine forest at $3,700 \sim 3800 \mathrm{~m}$ \\
\hline Gentiana grata Harry Sm. & Perennial herbs & Fl blue & Fl Aug.-Sept. & Alpine swamp at $2,900 \sim 4,050 \mathrm{~m}$ \\
\hline Gentiana linoides $\mathrm{Fr}$ & Annual herbs & Fl bluish-purple & Fl July-Aug. & Alpine shrubs and meadow at $3,000 \sim 4,000 \mathrm{~m}$ \\
\hline Gentiana maeulchanensis Fr & Annual herbs & Fl light blue & Fl Apr--July & Alpine shrubs at 2,500 3,600 m \\
\hline Gentiana ninglangensis TN Ho & Annual herbs & Fl light blue & Fl May & Alpine meadow at $2,500 \sim 3,000 \mathrm{~m}$ \\
\hline Gentiana pubiflora TN Ho & Annual herbs & Fl yellowish-green & Fl Apr--May & Alpine shrubs at $2,600 \sim 3,300 \mathrm{~m}$ \\
\hline Gentiana qiujiangensis $\mathrm{TN}$ Ho & Perennial herbs & Fl bluish-purple & Fl Aug. & Alpine meadow, alpine swamp at about $3,900 \mathrm{~m}$ \\
\hline Gentiana stellulata Harry Sm. & Annual herbs & Fl blue & Fl June-Sept. & Alpine meadow at $3,300 \sim 3,950 \mathrm{~m}$ \\
\hline Gentiana subintricata TN Ho & Annual herbs & Fl light purplish-red & Fl July-Aug. & Alpine meadow at $3,400 \sim 3,700 \mathrm{~m}$ \\
\hline Gentiana subtilis Harry Sm. & Annual herbs & Fl bluish & Fl Apr--July & Alpine meadow at $3,650 \sim 4,500 \mathrm{~m}$ \\
\hline Hedysarum limitaneum Hand.-Mazz. & Perennial herbs & Fl yellow & Fl July & Evergreen broad-leaved forest at $1,500 \sim 3,200 \mathrm{~m}$ \\
\hline Hemerocallis nana WW Sm. et Forr. & Small Perennial herbs & Fl orange yellow & Fl June & Subalpine or alpine shrubs at 2,400 3,400 m \\
\hline Herminium carnosilabre $\mathrm{T}$. Tang et FT Wang & Herbs & Fl brown & Fl May & Subalpine meadow at about $2,900 \mathrm{~m}$ \\
\hline Hypericum elliptifolium $\mathrm{HL} \mathrm{Li}$ & Shrubs & Fl light yellow & Fl Aug.-Sept. & Coniferous and broad-leaved mixed forest at $1,800 \sim 2,200 \mathrm{~m}$ \\
\hline Impatiens ceratophora Comber & Herbs & Fl white, pink, orange, red & Fl July-Oct. & Subalpine shrubs and meadow at $1,700 \sim 2,700 \mathrm{~m}$ \\
\hline Impatiens chimiliensis HF Comber & Herbs & Fl white, pink, orange, red & Fl July-Oct. & Subalpine shrubs and meadow at $3,200 \sim 3,600 \mathrm{~m}$ \\
\hline Impatiens microcentra Hand.-Mazz. & Herbs & Fl white, pink, orange, red & Fl July-Oct. & Subalpine meadow at $2,200 \sim 2,800 \mathrm{~m}$ \\
\hline Impatiens taronensis Hand.-Mazz. & Herbs & Fl white, pink, orange, red & Fl July-Oct. & Subalpine meadow at $2,800 \sim 3,100 \mathrm{~m}$ \\
\hline Impatiens tomentella JD Hooker & Herbs & Fl white, pink, orange, red & Fl July-Oct. & Evergreen broad-leaved forest at $1,400 \sim 1,800 \mathrm{~m}$ \\
\hline Impatiens xanthina $\mathrm{HF}$ Comber & Herbs & Fl white, pink, orange, red & Fl July-Oct. & Evergreen broad-leaved forest at $1,500 \sim 2,800 \mathrm{~m}$ \\
\hline Indigofera calcicola Craib & Shrubs & Fl purple-red & Fl Aug.-Oct. & Subalpine shrubs at 2,400 2,600 m \\
\hline Indigofera densa $\mathrm{NE} \mathrm{Br.}$ & Shrubs & Fl yellow & Fl June-July & Alpine rhododendron shrubs at 2,400 3,600 m \\
\hline Indigofera sensitiva $\mathrm{Fr}$ & Shrubs & Fl purple-red & Fl Aug.-Sept. & Subalpine shrubs at 2,200 2,600 m \\
\hline Iris subdichotoma YT Zhao & Perennial herbs & Fl purplish & Fl Aug.-Oct. & Alpine shrubs and meadow at 2,200 3,400 m \\
\hline Lilium habaense FT Wang et T. Tang & Perennial herbs & Fl yellowish-green & Fl July-Aug. & Alpine shrubs at $3,300 \mathrm{~m}$ \\
\hline Listera bambusetorum Hand.-Mazz. & Adnascent herbs & Fl pink & Fl May-June & Evergreen broad-leaved forest at $800 \sim 1,260 \mathrm{~m}$ \\
\hline Lysionotus angustisepalus WT Wang & Adnascent subshrubs & Fl purplish & Fl Sept. & Dry-hot valley shrubs at about $1,200 \mathrm{~m}$ \\
\hline Lysionotus gracilis WW Sm. & Subshrubs & Fl purplish & Fl July-Aug. & Evergreen broad-leaved forest at $2,000 \sim 2,250 \mathrm{~m}$ \\
\hline Lysionotus sessilifolius Hand.-Mazz. & Subshrubs & Fl light purple & Fl Aug.-Sept. & Evergreen broad-leaved forest at $1,250 \sim 2,800 \mathrm{~m}$ \\
\hline Lysionotus sulphureus Hand.-Mazz. & Subshrubs & Fl yellowish & Fl Sept. & Evergreen broad-leaved forest at $2,300 \sim 2,800 \mathrm{~m}$ \\
\hline Meconopsis delavayi (Fr) Fr ex Prain. & Perennial herbs & Fl deep purple & Fl May-July & Alpine meadow at $2,700 \sim 4,000 \mathrm{~m}$ \\
\hline Meconopsis Smithiana (Hand.-Mazz.) G. & Perennial herbs & Fl yellow & Fl June-July & Alpine meadow at $3,120 \sim 3,400 \mathrm{~m}$ \\
\hline Neillia serratisepala $\mathrm{HL} \mathrm{Li}$ & Shrubs & Fl white & Fl July-Aug. & Shrubs at $1,700 \sim 3,200 \mathrm{~m}$ \\
\hline Nomocharis aperta Wils. ex Fr & Perennial herbs & Fl yellowish & Fl June-July & Alpine shrubs at $2,800 \sim 4,000 \mathrm{~m}$ \\
\hline Nomocharis farreri (WE Evans) Harrow & Perennial herbs & Fl pink & Fl July & Alpine shrubs, meadow at $2,800 \sim 3,600 \mathrm{~m}$ \\
\hline Nomocharis forrestii Balf. & Perennial herbs & Fl pink & Fl June-July & $\begin{array}{l}\text { Coniferous and broad-leaved mixed forest, Rhododendron } \\
\text { shrubs, alpine shrubs at } 3,000 \sim 3,800 \mathrm{~m}\end{array}$ \\
\hline Nomocharis meleagrina $\mathrm{Fr}$ & Perennial herbs & Fl white & Fl June-July & $\begin{array}{l}\text { Deciduous broad-leaved forest, fir forest, alpine meadow, } \\
\text { shrubs at } 2,100 \sim 3,900 \mathrm{~m}\end{array}$ \\
\hline Olea laxiflora $\mathrm{HL} \mathrm{Li}$ & Evergreen shrubs & Fl white & Fl July-Aug. & Evergreen broad-leaved forest at about $900 \mathrm{~m}$ \\
\hline Omphalogramma elegans Forrest & Perennial herbs & Fl deep bluish-purple & Fl June-July & Alpine shrubs at $3,200 \sim 4,700 \mathrm{~m}$ \\
\hline Oreocharis aurantiaca Baill. & Perennial herbs & Fl purple-red & Fl Aug.-Sept. & Subalpine meadow at $1,700 \sim 3,000 \mathrm{~m}$ \\
\hline Paphiopedilum armeniacum Chen et Liu & Adnascent herbs & Fl yellow & Fl May-June & The wall of rock at 2,000 2,400 m \\
\hline Pedicularis atuntsiensis Bonati. & Perennial herbs & Fl purple & Fl July-Aug. & Alpine shrubs at 4,200 4,500 m \\
\hline Pedicularis crenularis $\mathrm{HL} \mathrm{Li}$ & Perennial herbs & Fl rose-red & Fl Oct. & Alpine fir forest at 2,400 3,000 m \\
\hline Pedicularis cyclorhyncha $\mathrm{HL} \mathrm{Li}$ & Perennial herbs & Fl purplish-red & Fl June & Alpine shrubs and meadow at $3,400 \sim 4,000 \mathrm{~m}$ \\
\hline Pedicularis dichrocephala Hand.-Mazz. & Perennial herbs & Fl purplish-red & Fl Aug. & Alpine shrubs and meadow at $3,200 \sim 3,500 \mathrm{~m}$ \\
\hline Pedicularis fastigiata $\mathrm{Fr}$ & Perennial herbs & Fl rose-red & Fl July & Alpine shrubs and meadow at $3,300 \sim 4,350 \mathrm{~m}$ \\
\hline Pedicularis filicula Fr ex Maxim. & Perennial herbs & Fl purplish-red & Fl May-July & Alpine shrubs and meadow at $2,800 \sim 4,880 \mathrm{~m}$ \\
\hline Pedicularis gracilicaulis $\mathrm{HL} \mathrm{Li}$ & Perennial herbs & Fl purplish-red & Fl July-Aug. & Alpine shrubs and meadow at 3,000 3,300 m \\
\hline Pedicularis habachanensis Bonati. & Perennial herbs & Fl red & Fl July & Alpine shrubs and meadow at $4,100 \sim 4,270 \mathrm{~m}$ \\
\hline Pedicularis humilis Bonati. & Perennial herbs & Fl rose-red & Fl July & Alpine shrubs and meadow at $3,050 \sim 4,000 \mathrm{~m}$ \\
\hline Pedicularis kariensis Bonati & Perennial herbs & Fl deep rose-red & Fl Aug.-Sept. & Alpine meadow at $2,400 \sim 4,000 \mathrm{~m}$ \\
\hline Pedicularis lamioides Hand.-Mazz. & Perennial herbs & Fl deep rose-red & Fl Aug.-Sept. & Alpine shrubs and meadow at $3,000 \sim 4,100 \mathrm{~m}$ \\
\hline Pedicularis lanpingensis HP Yang & Perennial herbs & Fl purplish-red & Fl June-July & Alpine shrubs and meadow at 3,200 3,500 m \\
\hline Pedicularis lecomtei Bonati, & Perennial herbs & Fl yellow & Fl June & Alpine meadow at $3,500 \sim 4,000 \mathrm{~m}$ \\
\hline Pedicularis macrorhyncha $\mathrm{HL} \mathrm{Li}$. & Perennial herbs & Fl purplish-red & Fl July-Sept. & Alpine meadow at $3,400 \sim 3,700 \mathrm{~m}$ \\
\hline Pedicularis maxonii Bonati, & Perennial herbs & Fl purple & Fl July & Alpine meadow at $2,500 \sim 3,000 \mathrm{~m}$ \\
\hline Pedicularis mayana Hand.-Mazz. & Perennial herbs & Fl purple & Fl May-Aug. & Alpine meadow at $3,700 \sim 4,400 \mathrm{~m}$ \\
\hline
\end{tabular}




\begin{tabular}{|c|c|c|c|c|}
\hline Species & Life form & Color & Fl or Fr time & Habitat \\
\hline Pedicularis meteororhyncha $\mathrm{HL} \mathrm{Li}$ & Perennial herbs & Fl purplish-red & Fl July-Aug. & Alpine meadow at $3,100 \sim 4,150 \mathrm{~m}$ \\
\hline Pedicularis micrantha $\mathrm{HL} \mathrm{Li}$ & Perennial herbs & Fl rose-red & Fl July & Alpine meadow at $3,100 \sim 4,400 \mathrm{~m}$ \\
\hline Pedicularis mussotii Fr & Perennial herbs & Fl red & Fl July-Aug. & Alpine meadow at $3,500 \sim 4,000 \mathrm{~m}$ \\
\hline Pedicularis obscura Bonati & Perennial herbs & Fl yellowish-red & Fl June & Alpine meadow at $3,600 \sim 4,200 \mathrm{~m}$ \\
\hline Pedicularis oligantha Fr ex Maxim. & Perennial herbs & Fl yellow & Fl July & Alpine meadow at $2,500 \sim 3,000 \mathrm{~m}$ \\
\hline Pedicularis orthocoryne $\mathrm{HL} \mathrm{Li}$ & Perennial herbs & Fl white-yellow & Fl June & Alpine meadow at $4,420 \sim 4,480 \mathrm{~m}$ \\
\hline Pedicularis pinetorum Hand.-Mazz. & Perennial herbs & Fl red & Fl Aug. & Subalpine meadow at $2,500 \sim 2,800 \mathrm{~m}$ \\
\hline Pedicularis praeruptorum Bonati. & Perennial herbs & Fl red & Fl June-Aug. & Alpine meadow at $3,600 \sim 4,400 \mathrm{~m}$ \\
\hline Pedicularis pseudoversicolor Hand.-Mazz. & Perennial herbs & Fl yellow & Fl May-June & Alpine meadow at $3,600 \sim 4,400 \mathrm{~m}$ \\
\hline Pedicularis remotiloba Hand.-Mazz. & Perennial herbs & Fl rose-red & Fl Aug. & Alpine meadow at $3,600 \sim 4,000 \mathrm{~m}$ \\
\hline Pedicularis salicifolia Bonati. & Perennial herbs & Fl deep rose-red & Fl July-May & Subalpine, alpine meadow at $1,000 \sim 3,500 \mathrm{~m}$ \\
\hline Pedicularis sigmoidea Fr ex Maxim. & Perennial herbs & Fl purple-red & Fl Aug. & Alpine meadow at $3,000 \sim 3,600 \mathrm{~m}$ \\
\hline Pedicularis tomentosa $\mathrm{Li}$ & Perennial herbs & Fl yellow & Fl Aug.-Sept. & Alpine meadow at 2,300 3,000 m \\
\hline Pedicularis tsaii $\mathrm{HL} \mathrm{Li}$ & Perennial herbs & Fl purple & Fl Aug. & Alpine meadow at $4,000 \sim 4,250 \mathrm{~m}$ \\
\hline Pedicularis umbelliformis $\mathrm{HL} \mathrm{Li}$ & Perennial herbs & Fl purple-red & Fl June-Aug. & Alpine meadow at $3,440 \sim 4,000 \mathrm{~m}$ \\
\hline Pedicularis weixiensis HP Yang & Perennial herbs & Fl purple & Fl June-July & Apine rhododendron shrubs at $3,600 \sim 4,000 \mathrm{~m}$ \\
\hline Pedicularis yui $\mathrm{HL} \mathrm{Li}$ & Perennial herbs & Fl purple & Fl June & Alpine rhododendron shrubs at $3,600 \sim 4,000 \mathrm{~m}$ \\
\hline Pedicularis zhongdianensis HP Yang & Perennial herbs & Fl purplish-red & Fl June-July & Alpine meadow at $3,300 \sim 4,300 \mathrm{~m}$ \\
\hline Photinia prionophylla $(\mathrm{Fr}) \mathrm{CK}$ Schneid & Shrubs & Fl white, Fr red & Fl May, Fr Sept.-Nov. & Dry-hot valley shrubs at $1,940 \sim 2,700 \mathrm{~m}$ \\
\hline Platanthera likiangensis FT Wang & Adnascent herbs & Fl yellowish & Fl July-Aug. & Evergreen broad-leaved forest at $800 \sim 2,800 \mathrm{~m}$ \\
\hline Platanthera sinica $\mathrm{T}$ Tang et FT Wang & Herbs & Fl greenish & Fl Aug.-Sept. & Alpine meadow at $3,200 \mathrm{~m}$ \\
\hline Pleione forrestii Schltr. & Herbs & Fl green & Fl July-Aug. & Alpine meadow at $1,950 \sim 3,900 \mathrm{~m}$ \\
\hline Potentilla delavayi $\mathrm{Fr}$ & Perennial herbs & Fl yellow & Fl July-Sept. & Subalpine shrubs and meadow at $3,000 \sim 4,200 \mathrm{~m}$ \\
\hline Primula annulata Balf. et Kingdon-Ward & Perennial herbs & Fl blue-purple & Fl July & The wall of limestone at $4,700 \mathrm{~m}$ \\
\hline Primula bullata $\mathrm{Fr}$ & Perennial herbs & Fl deep yellow & Fl May-June & Pine forest at $3,000 \mathrm{~m}$ \\
\hline Primula dryadifolia Fret. & Perennial herbs & Fl light red & F1 June-July & Alpine meadow at $4,000 \sim 5,500 \mathrm{~m}$ \\
\hline Primula euosma Craib & Perennial herbs & Fl deep purplish-blue & Fl Apr--July & Alpine meadow at about $3,000 \mathrm{~m}$ \\
\hline Primula forrestii Balf. & Perennial herbs & Fl deep gold-yellow & Fl Apr--May & Alpine shrubs at 3,000 3,200 m \\
\hline Primula runcinata $\mathrm{CM} \mathrm{Hu}$ & Perennial herbs & Fl blue & Fl July & Alpine shrubs at $3,100 \sim 3,200 \mathrm{~m}$ \\
\hline Primula sinolisteri Balf. & Perennial herbs & Fl light red & Fl Feb.-Aug. & $\begin{array}{l}\text { Alpine shrubs, coniferous and broad-leaved mixed } \\
\text { forest at } 2,300 \sim 3,000 \mathrm{~m}\end{array}$ \\
\hline Ranunculus nematolobus Hand.-Mazz. & Perennial herbs & Fl yellow & Fl July-Sept. & Shrubs and meadow at $2,500 \sim 2,900 \mathrm{~m}$ \\
\hline Rhodiola nobilis (Fret) SH Fu & Perennial herbs & Fl red, Fo pinks and reds & Fl May-Oct., Fo Aug.-Oct. & Alpine meadow at 2,500 4,200 m \\
\hline Rhododendron albertsenianum Forrest & Shrubs & Fl red to purple & Fl Apr--May & Coniferous and broad-leavedd mixed forest at $700 \sim 2,600 \mathrm{~m}$ \\
\hline Rhododendron araiophyllum Balf. et WW Sm. & Shrubs & Fl white & Fl May-June & Alpine fir forest, alpine rhododendron shrubs at $3,000 \sim 4,200 \mathrm{~m}$ \\
\hline Rhododendron basilicum Balf. et WW Sm. & Shrubs & Fl rose & Fl May-July & Alpine rhododendron shrubs at 3,900 4,200 m \\
\hline Rhododendron charitopes Balf. et Farrer. & Shrubs & Fl rose & Fl Apr. & Coniferous and broad-leavedd mixed forest at about $2,600 \mathrm{~m}$ \\
\hline Rhododendron chrysodoron Tagg ex Hutch. & Small shrubs & Fl pink & Fl May-July & Alpine rhododendron shrubs at $3,000 \sim 3,400 \mathrm{~m}$ \\
\hline Rhododendron codonanthum Balf. et Forrest & Groundling small shrubs & Fl deep red & Fl May-July & Alpine rhododendron shrubs at $3,400 \sim 4,100 \mathrm{~m}$ \\
\hline Rhododendron cyanocarpum ( Fr) WW Sm. & Shrubs & Fl deep red & Fl May-July & fir forest, alpine Rhododendron shrubs at 3,270 4,050 m \\
\hline Rhododendron dendricola Hutch. & Shrubs & Fl light yellow & F1 May-June & Coniferous and broad-leavedd mixed forest at $1,200 \sim 2,800 \mathrm{~m}$ \\
\hline Rhododendron dumicola Tagg et Forrest & Shrubs & Fl deep yellow & Fl May & Alpine rhododendron shrubs at 2,600 3,050 m \\
\hline Rhododendron facetum IB Balfour et Kingdon-Ward & Shrubs & Fl purple & Fl May-June & Alpine rhododendron shrubs, fir forest at 3,500 4,500 m \\
\hline Rhododendron fastigiatum $\mathrm{Fr}$ & Shrubs, small trees & Fl deep red & Fl Apr--May & Evergreen broad-leaved forest at $1,600 \sim 2,700 \mathrm{~m}$ \\
\hline $\begin{array}{l}\text { Rhododendron gemmiferum MN Philipson et } \\
\text { WR Philipson }\end{array}$ & Shrubs & Fl yellow or pink & Fl May-July & Alpine rhododendron shrubs, fir forest at 3,500 3,900 m \\
\hline Rhododendron gongshanense T. L. Ming & Shrubs & Fl reddish & Fl Apr--June & $\begin{array}{l}\text { Alpine rhododendron shrubs, evergreen broad-leaved } \\
\text { forest at } 1,900 \sim 3,600 \mathrm{~m}\end{array}$ \\
\hline Rhododendron haematodes $\mathrm{Fr}$ & Small shrubs & Fl purplish & Fl May-June & $\begin{array}{l}\text { Alpine rhododendron shrubs,alpine gravel desert at } \\
3,100 \sim 4,000 \mathrm{~m}\end{array}$ \\
\hline $\begin{array}{l}\text { Rhododendron kyawi Lace et WW Sm. } \\
\text { forest at 2,700 4,450 m }\end{array}$ & Shrubs, small trees & Fl white & Fl May-June & Alpine rhododendron shrubs, coniferous and broad-leaved mixed \\
\hline Rhododendron lateriflorum RC Fang et AL Chang & Deciduous shrubs & Fl pink or white & Fl Mar--May & Evergreen broad-leaved forest at $600 \sim 2,400 \mathrm{~m}$ \\
\hline Rhododendron leptopeplum Balf. f. et Forrest & Evergreen shrubs & Fl white & Fl May & Coniferous and broad-leaved mixed forest at 2,300 2,700 m \\
\hline $\begin{array}{l}\text { Rhododendron mollicomum Mollis. } \\
\text { forest at } 2,000 \sim 3,400 \mathrm{~m}\end{array}$ & Shrubs, small trees & Fl white to pink & Fl Apr--May & Evergreen broad-leaved forest, coniferous and broad-leaved mixed \\
\hline Rhododendron nakotiltum Balf. et Forrest & Shrubs, small trees & Fl white & Fl May-June & Alpine rhododendron shrubs, fir forest at $2,600 \sim 4,200 \mathrm{~m}$ \\
\hline Rhododendron polycladum $\mathrm{Fr}$ & Shrubs & Fl purplish-red & Fl May-June & Alpine rhododendron shrubs, fir forest at $3,200 \sim 4,500 \mathrm{~m}$ \\
\hline Rhododendron pronum Tagg et Forrest & Shrubs & Fl purplish-red & Fl May-June & Evergreen broad-leaved forest at 1,100 2,500 m \\
\hline Rhododendron rhombifolium RC Fang & Shrubs, small trees & Fl yellowish-green & Fl May-June & Alpine rhododendron shrubs, fir forest at $3,000 \sim 4,450 \mathrm{~m}$ \\
\hline Rhododendron rothschildii Davidian & Shrubs & Fl yellow & Fl May-June & Alpine rhododendron shrubs at 3,200 4,400 m \\
\hline Rhododendron rude Tagg et Forrest & Small shrubs & Fl yellow & Fl May & Alpine rhododendron shrubs at $2,600 \sim 2,800 \mathrm{~m}$ \\
\hline Rhododendron russatum Balf. et Forrest & Shrubs & Fl white & Fl May-June & Alpine meadow at $1,500 \sim 2,400 \mathrm{~m}$ \\
\hline Rhododendron sperabile IB Balfour et Farrer & Shrubs & Fl red & Fl Mar--July & Alpine rhododendron shrubs at $2,300 \sim 3,800 \mathrm{~m}$ \\
\hline Rhododendron taliense $\mathrm{Fr}$ & Shrubs & Fl red & Fl Apr--May & Coniferous and broad-leaved mixed forest at $1,850 \sim 2,100 \mathrm{~m}$ \\
\hline Rhododendron trichocladum Fret & Shrubs & Fl white or pink & Fl June-July & Alpine rhododendron shrubs, fir forest at 3,500 4,500 m \\
\hline Rhodoleia forrestii Chun ex Exell & Evergreen trees & Fl red & $\mathrm{Fl}$ after Oct. & Coniferous and broad-leaved mixed forest at $1,600 \sim 2,800 \mathrm{~m}$ \\
\hline Rosa glomerata Rehd. Et Wils. & Shrubs & Fl pink & Fl July-Sept. & Subalpine shrubs at 2,400 2,900 m \\
\hline Rosa praelucens Bijh. & Shrubs & Fl red & Fl June-July & Subalpine shrubs at 2,700 3,000 m \\
\hline Rosa taronensis TT Yü et TC Ku & Spined shrubs & Fl yellowish & Fl July-Aug. & Shrubs and meadow at $2,900 \sim 3,400 \mathrm{~m}$ \\
\hline Rosa weisiensis $\mathrm{Yu}$ et $\mathrm{Ku}$. & Climbling shrubs & Fl white & Fl Apr--May & Subalpine shrubs at $1,850 \sim 2,300 \mathrm{~m}$ \\
\hline Saussurea delavayi $\mathrm{Fr}$ & Herbs & Fl purple & Fl July-Sept. & Alpine gravel desert at $3,300 \sim 3,660 \mathrm{~m}$ \\
\hline Saussurea grosseserrata $\mathrm{Fr}$ & Perennial herbs & Fl yellow & Fl July-Sept. & Alpine gravel desert at $3,200 \sim 4,800 \mathrm{~m}$ \\
\hline Saussurea peduncularis $\mathrm{Fr}$ & Perennial herbs & Fl purple & Fl May-Sept. & Alpine gravel desert at $4,200 \sim 4,500 \mathrm{~m}$ \\
\hline Saussurea porphyroleuca Hand.-Mazz. & Perennial herbs & Fl purple & Fl Aug.-Sept. & Alpine meadow, alpine gravel desert at 3,800 4,800 m \\
\hline Saussurea pratensis Anthony & Perennial tufty herbs & Fl purplish-red & Fl July-Oct. & Alpine shrubs, alpine gravel desert at 3,500 4,500 m \\
\hline Saussurea rockii Anthony & Perennial cushion herbs & Fl light purple & Fl Aug.-Sept. & Alpine shrubs, alpine gravel desert at 3,500 4,500 m \\
\hline Saussurea semiamplexicaulis Lipschitz & Perennial herbs & Fl yellow & F1 July-Sept. & Alpine shrubs and meadow at 3,000 5,200 m \\
\hline Saussurea uliginosa Hand.-Mazz. & Perennial cushion herbs & Fl purplish-red & Fl July-Aug. & Alpine gravel desert at 4,500 5,200 m \\
\hline Sedum magniflorum KT Fu & Perennial herbs & Fl yellow & Fl June-Aug. & Alpine shrubs, meadow and gravel desert at 3,300 5,400 m \\
\hline Sedum wangii $\mathrm{SH} \mathrm{Fu}$ & Perennial herbs & Fl yellow & Fl May-Aug. & Coniferous and broad-leaved mixed forest at $1,300 \sim 2,700 \mathrm{~m}$ \\
\hline Silene atrocastanea Diels. & Perennial herbs & Fl purple & Fl Aug.- Nov. & Alpine shrubs and meadow at 2,900 3,600 m \\
\hline Silene chodatii Bocq. & Perennial herbs & Fl purple-brown & Fl Aug.- Nov. & Alpine shrubs and meadow at $2,700 \sim 3,800 \mathrm{~m}$ \\
\hline Silene delavayi $\mathrm{Fr}$ & Perennial herbs & Fl purple-red & Fl June-Nov. & Alpine shrubs and meadow at 2,700 3,000 m \\
\hline Silene lichingensis KT Fu & Perennial herbs & Flred & Fl July-Aug. & Alpine shrubs at $2,900 \sim 3,300 \mathrm{~m}$ \\
\hline
\end{tabular}




\begin{tabular}{|c|c|c|c|c|}
\hline Species & Life form & Color & Fl or Fr time & Habitat \\
\hline Silene lineariloba $\mathrm{CY} \mathrm{Wu}$ & Perennial herbs & Fl purple & Fl Aug.-Oct. & Alpine shrubs at $2,700 \sim 2,900 \mathrm{~m}$ \\
\hline Silene longipes (Post) D. & Perennial herbs & Fl purple & $\mathrm{Fl}$ after Aug. & Subalpine meadow, alpine shrubs at 3,300 4,000 m \\
\hline Silene melanantha Fret & Perennial herbs & F deep purple & Fl July-Sept. & Alpine shrubs and meadow at $3,200 \sim 4,150 \mathrm{~m}$ \\
\hline Silene otodonta $\mathrm{Fr}$ & Perennial herbs & Fl light red & Fl after Sept. & Subalpine meadow at $2,100 \sim 2,500 \mathrm{~m}$ \\
\hline Silene phoenicodonta $\mathrm{Fr}$ & Perennial herbs & Fl deep purple & Fl after July & Subalpine meadow at about $1,800 \mathrm{~m}$ \\
\hline Silene pubicalycina (Fenzl) Maire & Perennial herbs & Fl reddish & $\mathrm{Fl}$ after July & Alpine shrubs at about $3,200 \mathrm{~m}$ \\
\hline Silene rosiflora Kingdon-Ward & Perennial herbs & Fl reddish & Fl May-July & Alpine shrubs, alpine gravel desert at $1,900 \sim 2,600 \mathrm{~m}$ \\
\hline Silene scopulorum $\mathrm{Fr}$ & Perennial herbs & Fl dark purple & Fl July-Aug. & Alpine meadow, alpine gravel desert at about $4,000 \mathrm{~m}$ \\
\hline Silene yunnanensis Fret & Perennial herbs & Fl deep purple & Fl June-Sept. & Alpine shrubs, alpine gravel desert at 2,400 3,900 m \\
\hline $\begin{array}{l}\text { Smithorchis calceoliformis (WW Sm.) } \\
\text { T. Tang et FT Wang }\end{array}$ & Herbs & Fl white & Fl Dec. & Evergreen broad-leaved forest at $1,000 \sim 2,100 \mathrm{~m}$ \\
\hline Solms-Laubachia pulcherrima Muschler & Perennial shrublike herbs & Fl bluish, Fr purplish-red & Fl Apr--June, Fr July-Aug. & Alpine gravel desert at $3,400 \sim 4,300 \mathrm{~m}$ \\
\hline Sorbus harrowiana (IB Balfour et WW Sm.) Rehder & Trees & Fl white & Fl May-June & Evergreen broad-leaved forest at 2,100 3,500 m \\
\hline Sorbus kiukiangensis TT Yu & Shrubs or trees & Fl white, Fr red & Fl May-June & Evergreen broad-leaved forest at $1,700 \sim 3,000 \mathrm{~m}$ \\
\hline Sorbus poteriifolia Hand.-Mazz. & Small shrubs & Fl pinkish-white, Fr white & Fl May-June, Fr Sept.- Oct. & Shrubs at $3,000 \sim 4,000 \mathrm{~m}$ \\
\hline Spiraea calcicola WW Sm. & Shrubs & Fl white & Fl May-June & Subalpine shrubs and meadow at $2,700 \sim 2,800 \mathrm{~m}$ \\
\hline Spiraea compsophylla Hand.-Mazz. & Shrubs & Fl white & Fl July-Sept. & Shrubs and meadow at $1,450 \sim 2,450 \mathrm{~m}$ \\
\hline
\end{tabular}

Table 4. The relationships between endemic wild ornamental plants and their habitat.

\begin{tabular}{lcc}
\hline & $\begin{array}{c}\text { Endemic wild } \\
\text { ornamental } \\
\text { plants } \\
\text { (species) }\end{array}$ & Percentage \\
Habitat & 73 & 27.8 \\
\hline Alpine shrub & 70 & 26.6 \\
Alpine meadow & 29 & 11.0 \\
Rhododendron scrub & 28 & 10.6 \\
Evergreen broad-leaved forest & 27 & 10.3 \\
Alpine gravel desert & 20 & 7.6 \\
Subalpine or alpine meadow & 17 & 6.6 \\
Conifer and broad-leaved mixed forest & 5 & 1.9 \\
Dry-hot valley scrub & & \\
\hline
\end{tabular}

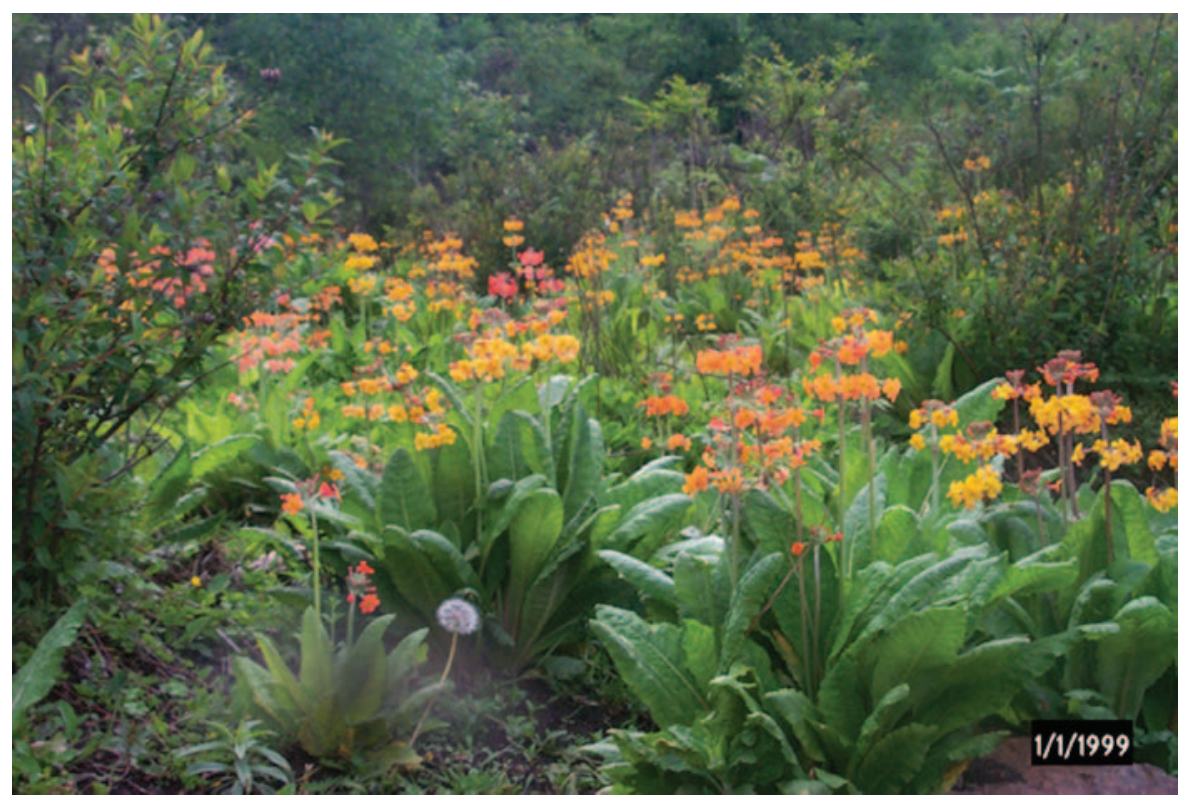

Fig. 2. Primula bulleyana Forr. Taken in Yulong snow Mountains, meadows, altitude 2,700 m, in Lijian, northwestern Yunnan.

separate when ripe and are dispersed by the wind, whereas Actinidia pilosula has golden yellow fruits and the fruit of Berberis spp. are often red in fall.

In terms of plants which have spectacular ornamental foliage, the inventory indicates that there are 15 species endemic to northwestern Yunnan. These species are dominated by several of the same families that also produce ornamental fruit, namely, Aceraceae, Rosaceae, and Anacardiaceae.

The habitats of the endemic wild flowers. The predominant habitats in the study sites are evergreen broad-leaved forest, subalpine or alpine meadow, alpine gravel desert, conifer and broad-leaved mixed forest, alpine scrub, Rhododendron scrub, and dry-hot valley scrub. The research shows that the endemic wild flowers are richest in the vegetation types of alpine scrub and alpine meadow, followed by alpine Rhododendron scrub, which means that endemic wild flowers are most abundant at elevations between 2,500 and $4,500 \mathrm{~m}$ (Table 4).

Subalpine meadow, alpine scrub and alpine gravel desert are special habitats for northwestern Yunnan. The Hengduan mountains are the consequence of the upheaval of the QinghaiXizang Plateau after the collision between the Indian plate and Eurasian plate (Wu, 1988), thus the vegetation types mentioned above were formed through rapid species differentiation. Alpine gravel desert is the high, cold desert plateau zone vegetation type, for which the typical ecological conditions are very low temperatures, a very short or nonexistent growing season, severe drought, high wind, and barren, rocky soil (Chang, 1981). In this harsh environment, plants formed adaptive physiological and ecological characteristics, developing into cushion plant communities Most of these cushion plants, small in size and peculiar in shape, grow in rock cracks, and their flowers are very bright and showy, thus they have excellent potential as rock gardens plants. For example, the genera Primula, Rhododendron, Meconopsis, Gentiana, and Lilium are all found in alpine gravel desert, with Primula and Meconopsis especially abundant in this habitat. In addition to these genera, many other alpine flowers, such as Aconitum spp., Delphinium spp., Corydalis spp., Draba spp., Solms-laubachia spp., Sedum spp., Rhodiola spp., Silene spp., and Saussurea spp. are also widely distributed in alpine gravel desert.

Subtropical evergreen broad-leaved forest only occurs at elevations of 2,300 to 3,000 $\mathrm{m}$ in northwestern Yunnan. This subtropical evergreen vegetation is the result of an unusual local environment, so it is not surprising that it has created a niche for many endemic wild ornamental plants. For example, subtropical conditions are present at 2,700 $\mathrm{m}$ in the Gaoligong mountains and the Dulong river valley due to the influence of the Indian warm-wet airstream.

Several rareornamental taxa with peculiar habitats. Interspersed with the forests are a number of wet meadows occurring on very gentle slopes where there is ground water seepage. Although species of Primula grow in variety of habitats, they often occur in great abundance and diversity in these moist meadows. One phenomenon in Primula (Fig. 2) is that two or more morphologically very similar species, but with totally different flower colors, often grow together. For example, $P$. forrestii with deep gold-yellow flowers, $P$. uncinata with blue flowers and $P$. sinolisteri with light red flowers often grow intermixed.

Another interesting habitat are the barren gravelly areas that occur in the saddles between peaks at elevations of $3,900 \mathrm{~m}$ to $4,600 \mathrm{~m}$. These gravely areas were formed by glacial deposits during past colder climates. These 
barren areas are exposed, windswept habitats, which suppresses the development of woody vegetation and limits the kinds of plants that can grow. Consequently, all plants in these areas are with stemless aboveground: the stems are either below ground, or the vegetative parts arise directly from subterranean bulbs, corms or rhizomes. In these exposed gravel habitats, the only parts of the plants visible above the ground are the leaves and flowers. Among the more common and conspicuous herbaceous plants that have evolved in these environments are Saussurea and Solms-Laubachia pulcherrima (Figs. 3 and 4). Saussurea species have interconnected underground stems with short branches that bear leaves and inflorescences

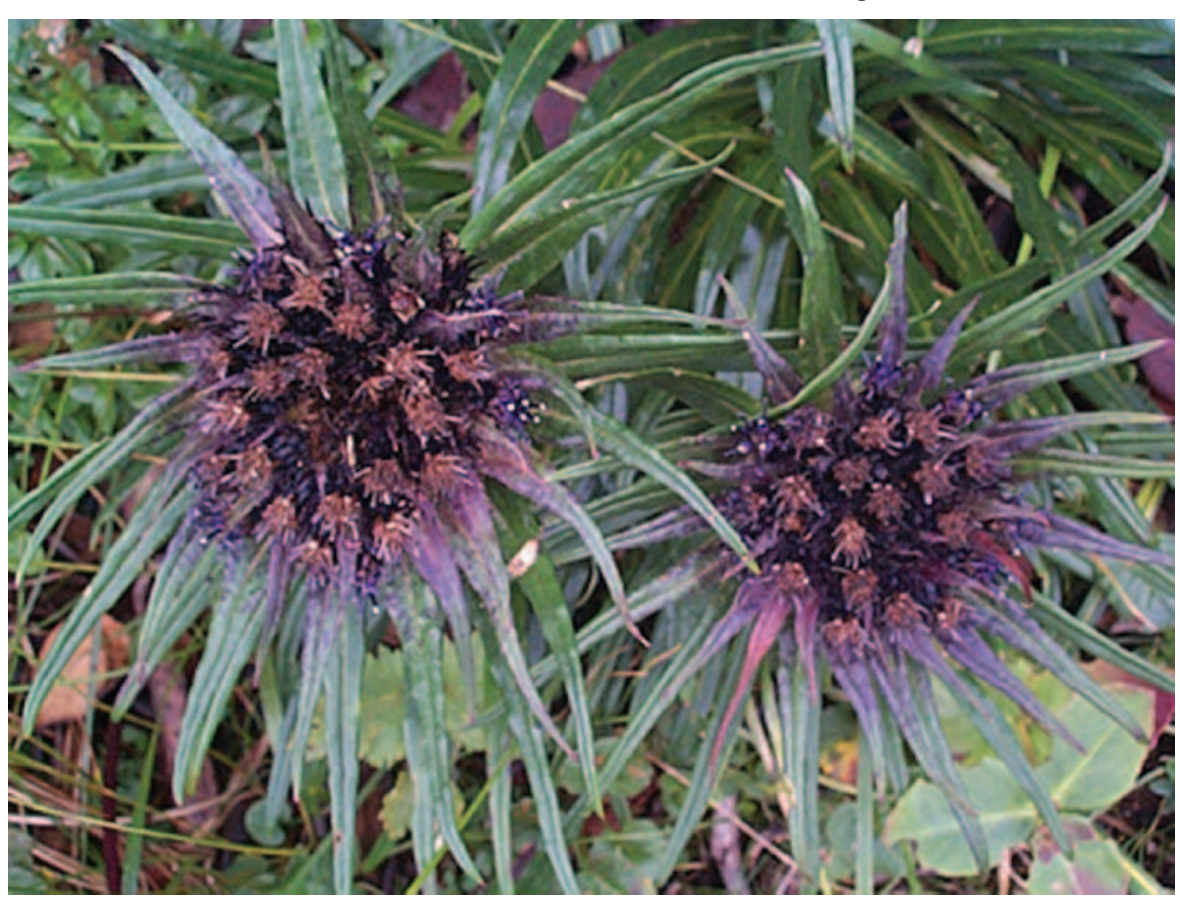

Fig. 3. Saussurea delavayi Fr. Taken in Daxueshan (big snow moutains, altitude 4,100 m), Zhongdian, Yunnan.

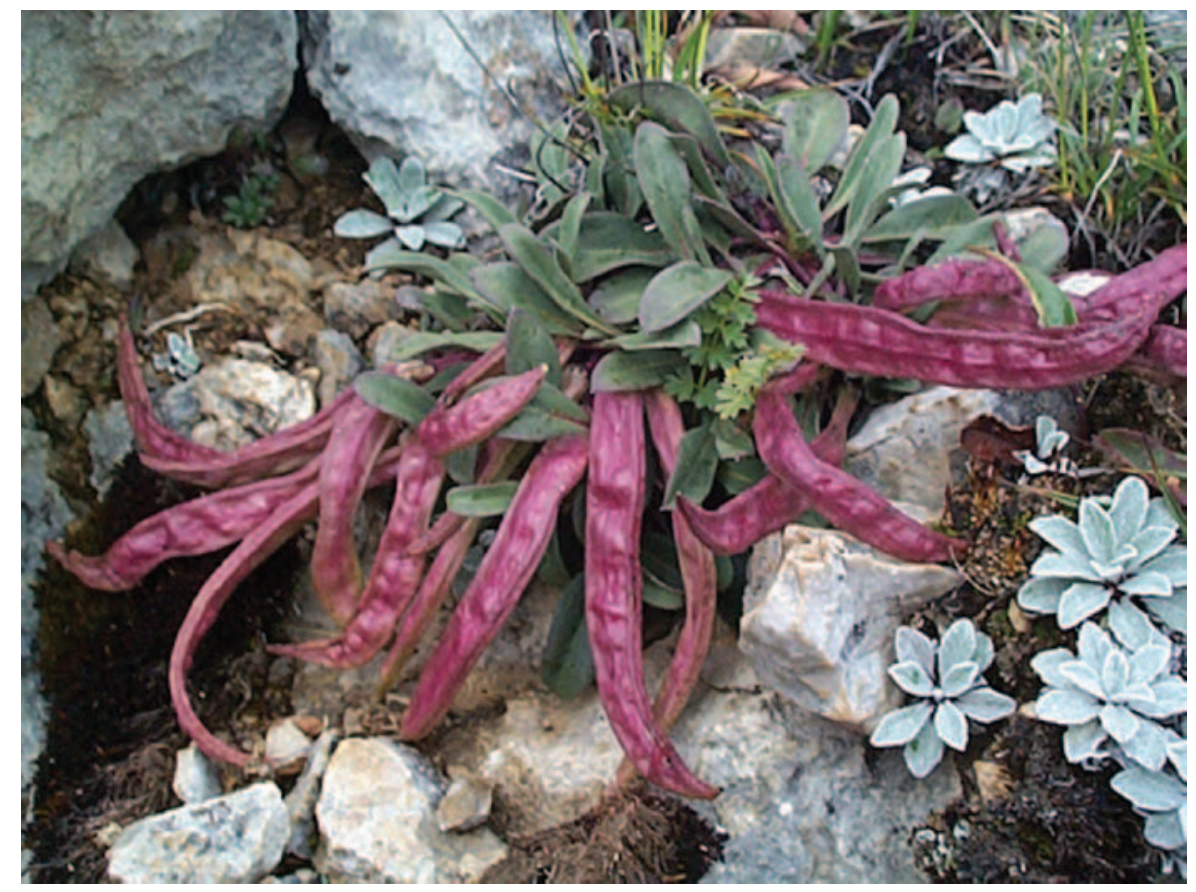

Fig. 4. Solms-Laubachia pulcherrima Muschler. Taken in Daxueshan (big snow moutains, altitude 4,100 m), in Zhongdian, Yunnan.

at the soil surface. What appears to be a small colony of several individual plants is actually a single plant with a turf of leaves at the end of each branch and inflorescences clustered in the center of the leaves. Similarly, SolmsLaubachia pulcherrima have the main stem and branches totally below ground level and only the leaves, flowers and fruits appear above the soil surface.

In contrast to the compact and low habit of other species in exposed environments, members of the genus Meconopsis (a member of the poppy family, Papaveraceae) often send their flowering stems well above the associated vegetation in defiance of the wind. This genus is characteristic of high elevation areas in north- western Yunnan and its habit in these exposed sites is rather amazing, since the flowers of Meconopsis appear to be extremely delicate. One important species, Meconopsis delavayi (Fig. 5), is grown for its beautiful flowers. The small, cup-shaped, pendant, lavender-blue flowers are borne singly on long, thin stems, in late spring and early summer, however, the heights of stems differ considerably among the varieties, ranging from 0.3 to $2 \mathrm{~m}$.

Another example of a genus characteristic of northwestern Yunnan is Gentiana (Fig. 6). This genus tends to be particularly abundant in alpine meadows at altitudes of $4,500 \mathrm{~m}$ to $4,700 \mathrm{~m}$. Of special interest to botanists in northwestern Yunnan are Gentianas that are annual or biennial herbs as their flowers are very colorful, ranging from dark red, purple, light yellow, green, white, and even to brightblue which is especially uncommon. Therefore, this genus is considered to have great potential for the future development of ornamental wildflower species.

\section{Conclusions}

As an initial regional ethnobotanical study of this area, it is clear that northwestern Yunnan is rich in endemic ornamental species. During the course of this study, we identified 262 taxa, belonging to 64 genera and 28 families, endemic to this region with potential ornamental value. This high number of endemic species reflects the diverse environment and taxonomic significance of northwestern Yunnan. Encouraging the use of natural ornamental plants in these regions can make considerable contributions to the economy and the culture of these rural regions. Therefore, the taxonomic investigation of these plants and the use of them by gardeners should be encouraged. These wild ornamental plants in northwest Yunnan not only are the ancestors for existing cultivated plants, but also are the rare basis for the cultivation of various new breeds in the future, as well as they are very important genetic resources, which could be used for introducing and conserving plants from the field. We hope that this work will help the researchers and people who are interested in wild ornamental plants.

\section{Literature Cited}

Chinese Academy of Science (Qinghai-Tibet Synthetic Scientific Expeditions teams). 1993, 1994. Vascular plants in Hengduan mountains. vols. 1 and 2. Science Press, Beijing, China.

Chang, D.H.S. 1981. The vegetation zonation of the Tibetan Plateau. Mountain Res. Dev. 1(1):29-48.

Chen, F.H. and Q.M. Hu. 1989. Flora of China, p. 1-248. vol. 59 (1). Science Press, Beijing, China.

Fang, Z.D. 1993. The wild flowers of Hengduan mountains in Yunnan China. Yunnan People Press, Kunming, China.

Feng, G.M. 1983. The Rhododendron in Yunnan. Yunnan People Press, Kunming, China.

Guan, K.Y., Z.K. Zhou, H. Sun, Y. Fei, and W.B. Sun. 1998. Highland flowers of Yunnan, p. 1-236. Science Press, Kunming, China.

Hu, Q.M. 1990. Flora of China. vol. 59(2). Science Press, Beijing, China. 


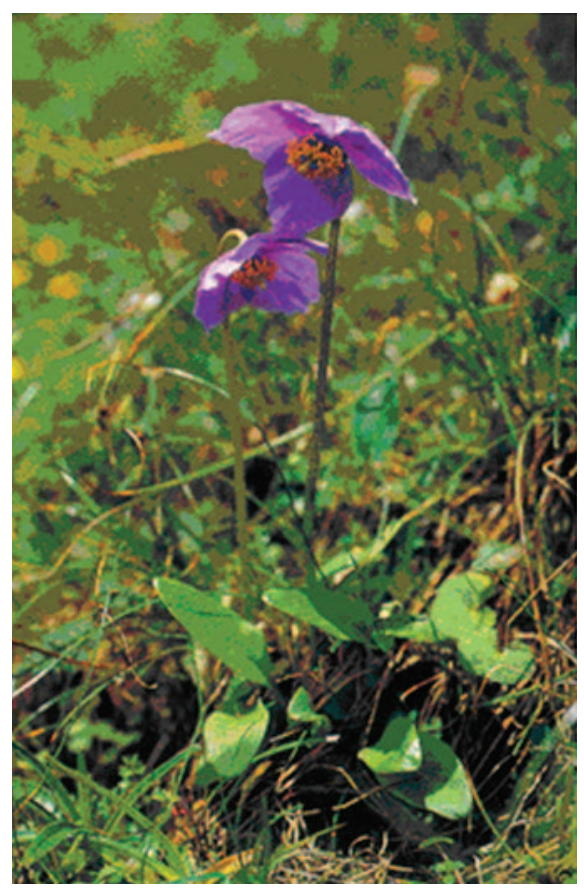

Fig. 5. Meconopsis delavayi (Fr) Fr ex Prain. Taken in Yulong snow Mountains, alpine grasslands at alt. 3,800 m, in Lijian, northwestern Yunnan.

Kunming Institute of Botany (Chinese Academic of Science). 1984. List of seed plants in Yunan. vols. 1 and 2. Yunnan People Press, Kunming, China.

Li, X.X., W.Y. Chen, K.Y. Guan, and Z.K. Zhou. 2003. A report on the wild ornamental plants from northwest Yunnan. Acta Bot. Yunnanica 25(4):435-446

Li, X.W. 1994. Two big biodiversity centers of Chinese endemic genera of seed plants and their characteristics in Yunnan province. Acta Bot. Yunnanica 16(3):221-227.

Li, X.W. and J.Li. 1993. A preliminary floristic study on the seed plants from the region of Hengduan mountain. Acta Bot. Yunnanica 15(3):217-213 Mittermeier, R.A., P.R. Gil, M. Hoffman, J. Pilgrim, T. Brooks, C.G. Mittermeier, J. Lamoreux, and G.A.B. da Fonseca. 2004. Hotspots revisited. CEMEX, Mexico City, México.

Shi, Z. and S.Y. Jin. 1999. Flora of China. vol. 78(2). Science Press, Beijing, China.
Sun, H. 2002. Tethys retreat and Himalayas-Hengduanshan Mountains uplift and their significance on the origin and development of the SinoHimalayan element and alpine flora. Acta Bot. Yunnanica 24(3):273-288

Wu, Z.Y. and S.K. Chen. 1986. Flora of Yunnan, p. 206-227, 336-602. vol. 4. Science Press, Beijing, China.

Wu, Z.Y. and Y.C. Zhu. 1987. Vegetation of Yunnan. Science Press, Beijing, China.

Wu, Z.Y. and S.K. Chen. 2000. Flora of Yunnan, p. 29-290, 538-695, 301-390. vol. 11. Science Press, Beijing, China.

Wu, Z.Y., S.K. Chen, and J. Chen. 1997. Flora of Yunnan, p.1-90, 640-824, 357-640. vol.7. Science Press, Beijing, China.

Wu, Z.Y. and T.Y. Ding. 1998. CD of the database of seed plants in China. Science Press, Kunming, Yunnan, China.

Wu, Z.Y. 1988. Hengduan mountain flora and her significance. J. Jpn. Bot. 63:297-311.

Yang, H.B. 1956. Flora of China. Vol.68. Science Press, Beijing, China.

Yang, Y.G. 1990. Physicogeographical regionalization of Yunnan. Higher Education Press, Beijing, China.

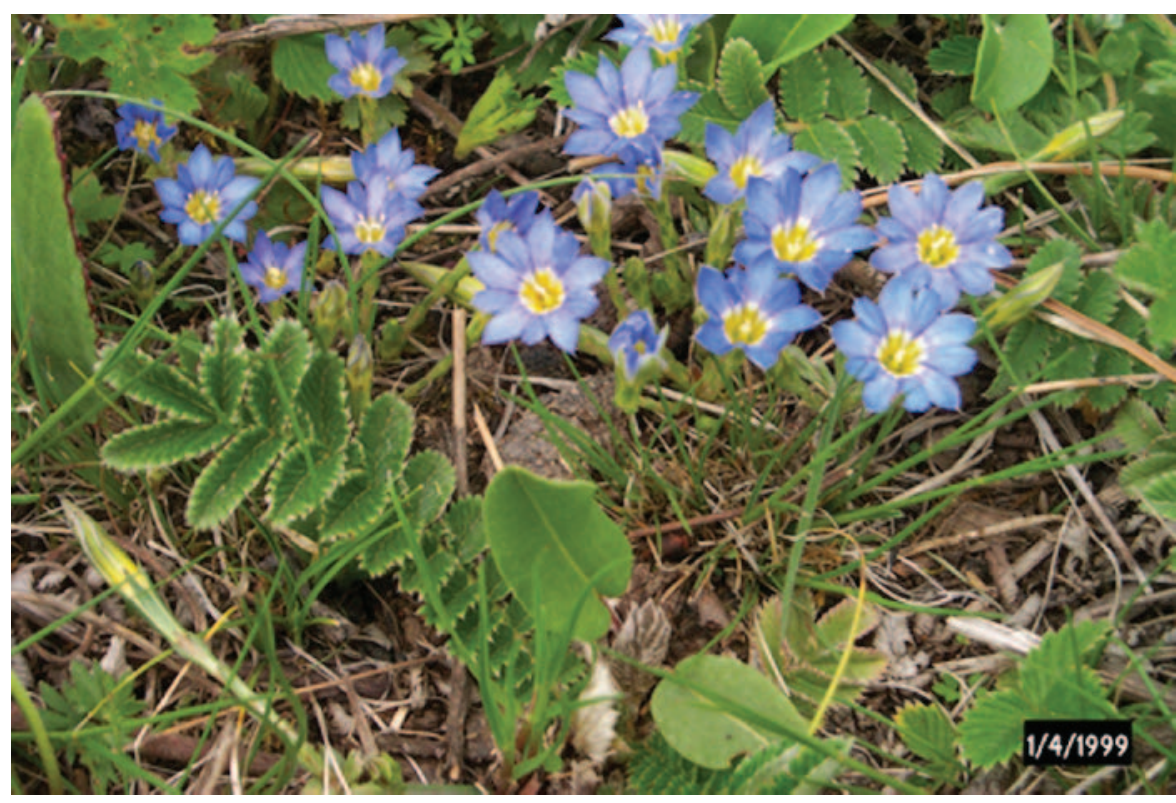

Fig. 6. Gentiana chungtienensis C. Marquand. Taken in alpine meadows, altitude 3,200 m, Xiaozhongdian, Yunnan. 